\title{
BMJ Open Self-harm and social media: thematic analysis of images posted on three social media sites
}

\author{
Nicola Shanahan, Cathy Brennan, ${ }^{\circledR}$ Allan House ${ }^{\odot}$
}

To cite: Shanahan N, Brennan C, House A. Selfharm and social media: thematic analysis of images posted on three social media sites. BMJ Open 2019:9:e027006. doi:10.1136/ bmjopen-2018-027006

- Prepublication history and additional material for this paper are available online. To view these files, please visit the journal online (http://dx.doi org/10.1136/bmjopen-2018027006).

Received 2 0ctober 2018 Revised 12 December 2018 Accepted 13 December 2018

D) Check for updates

(c) Author(s) (or their employer(s)) 2019. Re-use permitted under CC BY-NC. No commercial re-use. See rights and permissions. Published by BMJ.

Division of Psychological and Social Medicine, University of Leeds, Leeds, UK

Correspondence to Dr Cathy Brennan; C.A.Brennan@leeds.ac.uk

\begin{abstract}
Objectives To explore the nature of images tagged as self-harm on popular social media sites and what this might tell us about how these sites are used.

Design A visual content and thematic analysis of a sample of 602 images captured from Twitter, Instagram and Tumblr.

Results Over half the images tagged as self-harm had no explicit representation of self-harm. Where there was explicit representation, self-injury was the most common; none of these portrayed images of graphic or shocking self-injury. None of the images we captured specifically encouraged self-harm or suicide and there was no image that could be construed as sensationalising self-harm. Four themes were found across the images: communicating distress, addiction and recovery, gender and the female body, identity and belonging.

Conclusions Findings suggest that clinicians should not be overly anxious about what is being posted on social media. Although we found a very few posts suggesting self-injury was attractive, there were no posts that could be viewed as actively encouraging others to self-harm. Rather, the sites were being used to express difficult emotions in a variety of creative ways, offering inspiration to others through the form of texts or shared messages about recovery.
\end{abstract}

\section{INTRODUCTION}

Self-harm is a global public health challenge. Non-fatal repetition is common ${ }^{1}$ and self-harm is a strong predictor of completed suicide $^{2}$ and premature death. ${ }^{3}$ The UK has one of the highest rates of self-harm in Europe. ${ }^{4}$ In the UK self-harm accounts for over 150000 admissions to accident and emergency per year. ${ }^{5}$ Community-based studies suggest that up to $15 \%$ of adolescents have engaged in self-harm at some time, with lifetime prevalence higher for females than males. ${ }^{6}$

Studies have suggested that the average onset age of self-harming behaviours is in early adolescence. ${ }^{7}$ Although women selfharm more often than men, ${ }^{2} 8$ gender differences are not as great as is often supposed and about $40 \%$ of health service contacts after self-harm are male. ${ }^{9}$ The most striking

\section{Strengths and limitations of this study}

- First study to explore images tagged as self-harm across social media sites.

- Used established frameworks for the interpretation of visual imagery.

The rapid change of content on social media means our sample may not be representative of all images posted.

- Sampling images means we have no detail of those who are posting.

gender finding is the predominance of girls in the 13-16 age range, a pattern that has attracted recent attention in the UK because self-harm (or at least presentations following self-harm) have increased rapidly in this age group in recent years. ${ }^{1}$

Only a minority seek medical help following a self-harm episode, ${ }^{10}$ so there is a need to understand more about informal helpseeking. Since social media are widely used by the young, they have potential as a resource. However, social media are often discussed not as a helpful resource but as one of the reasons for an increase in rates of self-harm, particularly among adolescents. Academics, clinicians and others have expressed concerns about whether social media encourage self-harm, either through normalising the behaviour or through connecting young people with others who engage in it and encourage it. ${ }^{1112}$

Although a research literature is emerging in this area it is clear that we do not know much about what is being posted on social media about self-harm and why it is being posted. A key feature of social media is the sharing of images, photographs and memes through which online identities are constructed. ${ }^{13}$ In this study we therefore explored popular social media sites, to seek to understand what images were being posted and why-particularly to consider what cause for concern there might be about whether 
social media use increases the likelihood of self-harm. For example, by encouraging others or creating a desirable identity related to self-harm.

Although intentional self-harm (self-poisoning, self-injury) is usually differentiated in the literature from unintended self-harm such as might arise from an eating disorder or hazardous drinking, for this study we were interested in how the term was used in non-professional discourse and therefore studied all images tagged as selfharm on the social media sites we included.

\section{METHOD}

\section{Setting and sample}

We sampled images from three social media sitesTwitter, Instagram and Tumblr-chosen because they are three of the most popular social media sites that allow for image sharing.

Images and text incorporated in images were included in the study if they were tagged as self-harm, regardless of actual content. For example, the images did not have to show an act of self-harm and the text did not have to refer explicitly to self-harm, as long as the posting was tagged self-harm.

A priori exclusion criteria were (1) images obviously from an organisational account such as a mental health charity, because we wanted to be sure as far as possible that all images were posted by individuals rather than reflecting professional attempts to represent self-harm and (2) pornographic images-because of a decision that the impact on the researchers of viewing such images, and the impact on the project of work to obtain the extra approvals needed to be allowed to do so, would not be justified by the relevance of any findings to the main aim of the project.

Images were accessed by conducting a search of each site via the site's search function, using the tag of selfharm on each site (commonly using the hashtag system, \#). Each site was searched on one day in 2016 (chosen by NS) and from each of the three sites the 200 most recent images that fitted the inclusion criteria were included in the study. Due to a counting error that led to two extra images being collected from one site, a total of 602 images was analysed for this study.

\section{Patient and public involvement statement}

There was no patient/public involvement in this study.

\section{Analysis}

Images from each site were analysed in two ways, first descriptively for literal visual content and then for thematic content, according to the nature of the communicative function of the post.

\section{Visual content analysis procedure}

An initial coding frame was developed, informed by the available literature and research questions, which was built on deductively throughout the analytic process. This included categories on: the type of image, the subject of the image, gender in the image (if a person), representation of self-harm (including method, severity and body part), stated purpose and stated emotion (see online supplementary files for full framework).

Because of particular concerns about self-harm being portrayed positively in social media, we sought specifically three types of positive portrayal (1) text included as part of an image, that commented on self-harm as in some way pleasurable, desirable, or attractive (2) text included as part of an image, that expressly encouraged viewers to consider or to try self-harm (3) images without text, where a direct representation of self-harm (eg, a wound or a picture of somebody cutting) could be viewed as indicating a degree of glamour or desirability, or alternatively as being attractively transgressive. This third category clearly involved more judgement than the other two and decisions were therefore made only when all three authors were in agreement.

Each image was analysed individually for content. Once a full site had been analysed for content, the number of each occurrence of characteristics was noted before moving to the next site.

\section{Thematic analysis procedure}

We used a previously reported framework for analysing journalistic images to support the analysis of thematic content of the images. ${ }^{14}$ Categories included: content, captions, photo-techniques, emotions/feelings, symbols and visual rhetoric, intertextuality, message (see online supplementary files for full coding framework). Thematic analysis involved the following stages: familiarisation with the data; generating initial codes, approached with the research questions in mind; searching for themes; reviewing themes; defining and naming themes; and producing the report. ${ }^{15}$

Each image was analysed individually and then themes were derived by reviewing across cases and within social media site. Following each site analysis, a thematic map was developed.

Finally, a cross site analysis was completed exploring the similarities and differences across the three sites alongside any new themes.

The initial analysis was led by NS, although all authors contributed to the development of the themes through cross-checking with images and discussion of emerging analysis.

All images were classified as being within the public domain so were not subject to consent from individual posters. Images were stored on an encrypted server which only the lead researcher could access.

Due to copyright restrictions, we are unable to reproduce the images used in the analyses presented here. Similar examples are readily available by searching social media sites using the $<$ self-harm $>$ Tag function.

\section{RESULTS}

Visual content

In the first 602 images viewed we found none that met our exclusion criteria. 
An initial striking observation was the many ways in which self-harm was represented visually. Just over half $(325,54 \%)$ of the images did not explicitly represent the act of self-harm. Rather, they portrayed a variety of images including selfie shots $(116,19 \%)$, drawings, photographs of objects $(37,6 \%)$, references to films and memes. Images would also include text images that presented a certain mood, feeling or authored quote. A third of posts $(204,34 \%)$ contained no representation of a human form at all. Where people were represented there was a much higher proportion of females than males (199, 33\% of all images compared with $57,9.5 \%$ males).

The images that did portray self-harm predominantly displayed self-injury $(176,29 \%)$, nearly all cutting. Other forms of self-harm included eating disorders, bruising, scratching and substance use. When self-injury was depicted it was commonly on the arm or leg $(126,21 \%)$ and portrayed self-injury that was relatively non-severe. We found no really graphic or shocking photographs of severe mutilation-for example, showing exposed muscle or bone-although $37(6 \%)$ images were rated as severe-showing bleeding for example, or a wound that looked as if it might require medical treatment.

Identifying stated purpose and tone was difficult as often images were ambiguous. Nonetheless representations of distress were clear across all sites. Commonly images were posted to provide an understanding about the distress that was being experienced by the poster (213, $35 \%)$. There were other messages in the images, such as to inform others, reach out and share recovery $(46,8 \%)$. Sadness was the most easily identifiable emotional tone; anger, hope, loneliness and feeling overwhelmed were also identified.

Posters used a variety of additional tags to express their feelings, including a mix of mental health tags linking selfharm to terms like depression, eating disorders, sadness, suicide and anxiety.

None of the images we captured specifically encouraged self-harm or suicide. There was also no image that could be construed as sensationalising self-harm.

A small number of posts $(8,1.3 \%)$ incorporated text that implied self-harm was positive.

"That feeling of calm that you get after self-harm ... is like getting high ..."

[of scars] "I think they're cute"

\section{Thematic content}

There were four common themes identified across the three social media platforms. There were also themes that were specific to individual platforms.

\section{Communicating distress}

As discussed in the content analysis, sharing feelings was a common reason for posting, typically to communicate the distress experienced by the poster. This theme was the most prominent on Tumblr but all sites presented images where the poster communicated distress and sad feelings.
Posters would often incorporate quotes to articulate their distress-either written themselves or copied from others. These quotes expressed low self-worth, not feeling good enough, poor relationships with self or experiencing overwhelming feelings. Interestingly this theme was commonly presented through the medium of text in images which suggests that these are difficult emotions to articulate through imagery. An example of this was a black image incorporating the text: "I am just another nothing."

Interpersonal distress was also common-notes about how others had treated the poster, disappointing them, betraying them or letting them down. Loneliness and an inability to trust others featured in these posts. A drawn image of a face also contained the text: "Nothing hurts more than being disappointed by the single person you thought would never hurt you."

\section{Addiction and recovery}

Across all sites there were allusions to addiction and recovery. The language used in these images tended to describe addiction to self-harm in a similar way to other addictions. Posters described being 'clean' from selfharm or would post about how they felt 'called' to engage in self-harm. Sometimes posters were choosing social media sites to memorialise their recovery, perhaps in order to influence or support others, alongside perhaps keeping themselves in check and ensuring that they did not succumb again.

An example of an image from this theme is a beforeand-after shot of an arm. The before shot showed numerous cuts and scars, and the after shot showed that the scars had healed and faded. There were a number of these images across the three sites. These 'fading scar' images could be viewed as the poster feeling 'cleaner' now that the evidence of their self-harm is fading: their skin is recovering in the same way that they are. Another example of images in this theme is presentation of the number of days the poster had not engaged in self-harm.

\section{The presentation of gender and the female body}

We noted several themes related to the presentation of women, including visual depictions where gender was a striking feature, thinness was presented as a desirable characteristic, or women were presented as sexual or glamorous people. These images of thin, idealised and sexualised bodies, conforming to what is typically attractive, were countered by posts that seemed to subvert conventional ideas of glamour.

In the representation of gender, images were often sexually ambiguous or represented hybrid or hermaphrodite sexes. Even when there were pictures of men they were not as clearly 'masculine' as the pictures of women were 'feminine'. An image coded in this way was of a drawn cartoon like picture of Kylo Ren that had an obvious penis and also well-developed breasts-the image made reference to intersexuality and gender fluidity in text written with the image. 
When female bodies were shown in images, they were often slim and could be described as typically attractive by western standards. Occasionally thinness was actively noted and approved of-for example, in an image of a young woman lifting her top to show her torso, or another clasping her hands around a thigh to demonstrate its small girth.

Similarly, there were sexualised and glamorised images shown of women across all social media sites. Examples included a woman in lacy underwear lying on a bed, a cropped image of a woman lying in the bath strewn with petals, and women dressed glamorously but not in a social setting. Even so there was little nudity and only one breast in all the images we viewed-probably because all three sites are moderated to remove potentially sexual messaging.

Finally, there were images that subverted glamour. These images showed sexualised pictures of women which were subverted from typically western attractiveness. An example of this is women presented in dirty or ripped underwear, or masculine clothing. For example, one image we coded as subverted glamour was of legs in a model's pose but wearing badly ripped and bloodied tights.

\section{Identity and belonging}

There were messages of belonging throughout the data-set. Posters used tags with counter culture references to state that they 'belonged' to a different sub-culture (eg, \#BMTH, \#EMO, \#secretsociety123, \#goth) alongside hashtags where posters appeared to be referencing their mental health difficulties (\#anxiety, \#BPD, \#depression). In addition, some posts referred to self-harm in association with other behaviours (such as eating disorders) or other experiences (such as being bullied) which suggested a sense of shared belonging. Many posters also captioned their images with discussion points or questions suggesting that one of the functions was to connect with others who perhaps shared their experiences.

There were a number of images under this theme that referenced anime and manga culture; one example of these type of images was a manga style drawn picture in shades of blue and pink of two young girls in skimpy uniforms, one holding a knife and the other a blade. Both girls had noticeable scars on their thighs and there was an obvious sexualised aspect to the image in their positioning. These types of images were the only ones that could be interpreted as showcasing self-harm in a positive light.

In opposition to this there were also messages from posters stating that they felt excluded from society and that they did not feel they had a place anywhere.

\section{Differences between sites}

Although there were many similarities across all three sites, we noted that Twitter was different from Tumblr and Instagram in two respects. The images on Twitter presented more of an interactional element with images used to encourage others to act or express an opinion. There were also images on Twitter that were not observed on Instagram and Tumblr, tagged as self-harm but representing social issues such as 'Brexit'. Especially in this theme that we labelled 'Appropriation'-that is, use of the name of one type of behaviour to describe a different more social phenomenon. We also noted more use of humour on Twitter.

\section{DISCUSSION}

\section{Summary}

The results of our study suggest that social media are being used widely to represent self-harm in a variety of ways-a mix of photography of direct self-harm, text images, photographs of people, photographs of objects, selfies, collages, stock photos and creations such as drawings or cartoons. These images are not just depicting the act of self-harm in whatever form but also, among other things, motivations, distress, humour and ways of coping. From the images in our sample, there was no evidence of self-harm being sensationalised or that posters were using social media to encourage others to self-harm.

The images that did portray self-harm predominantly displayed self-injury, most notably cutting. Other studies on self-harm and social media have also found that cutting is most common form of self-harm presented. ${ }^{16} 17$ However, this should not lead to the conclusion that it is the most common type of self-harm. ${ }^{4}$ We also know that individuals use multiple methods across different episodes. ${ }^{18}$

There was a clear preponderance of female images where gender was depicted. Women are more frequent users of social media for personal posts, and younger women have higher rates of self-harm, but this female preponderance cannot be explained by markedly higher rates of self-injury among women. ${ }^{19}{ }^{20}$ However, many posts were not images of people and not all the images of people were images of the poster themselves so it is difficult to draw conclusions about who is posting on social media.

\section{Findings related to literature}

Our results suggested social media sites were used by those who engage in self-harm to share their experiences and feelings in a variety of ways. Not surprisingly, much of this communication involved straightforward statements and representations of distress, loneliness and concern about what the poster was doing.

There was also a visual and textual narrative in the analysis that self-harm is viewed as an addiction. There was a longing expressed through the images about the act of self-injury in particular that suggested some of the motivation for self-injury may be driven by a positive draw to repeat, an observation we link to our previous research identifying often overlooked positive functions of self-harm. ${ }^{21}$ 
Nixon et al explored addictive symptoms of self-injury in a sample of adolescents in hospital. Participants reported daily self-harm urges that did not always correspond with the act, and noted features of their self-injury that were consistent with addictive behaviour (upsetting but not enough to stop, frequency increasing). ${ }^{22}$ Self-injury is known to be related to other addiction-like behaviours such as drug abuse, disordered eating and sexual compulsivity. ${ }^{23}$

We noted the use of sexualised images of females, particularly on Tumblr and Instagram.

It could be that posters were sexualising self-harm specifically, or it could be that this is how they commonly post on all topics. Motz describes how women locate their sense of identity in their bodies so that any anger, contempt, shame or distress is expressed through self-injury, symbolising psychological pain. She suggests that there may be a sexual component to self-harm, the act itself providing a release from tension similar to that of an orgasm. ${ }^{24}$

It could be argued that the sexualisation of these images goes hand in hand with the gendered nature of the images. In mass media it tends to be the female body that is objectified ${ }^{25}$ so we would expect social media will be a reflection of the same trend. Young women feel pressure to present themselves as physically attractive as well as being rewarded for sexualised photographs and messages of promiscuity. ${ }^{26}$ Thus, the sexualised images posted in this study could have been posted as a response to the pressures the posters felt in conforming to presenting themselves in sexualised and attractive ways and were not specific to self-harm but represented a more general disposition to sexualisation of the female body across all media. $^{27}$

Themes of identity and belonging were common in the data. Indeed, connection with others is a commonly cited reason for accessing social media. ${ }^{28}$ It could be argued that posters are using social media sites as a way to develop their identity, using self-harm to try out various 'selves'. ${ }^{29}$ It has been suggested that with increasing technological changes (the use of smartphones and digital photography) the meaning of taking photographs has changed from an act of memory and retaining family heritage to becoming a tool for both identity formation and for personal communication. ${ }^{30}$ The results from this study suggest that alongside posting images to document posters' lives, the images also presented ways in which posters were testing out their identity and their sense of self-to develop an understanding of where they fitted within online communities. While this should not be seen as necessarily problematic, it may be that this search for belonging is an incentive to identify with self-harm, as a ticket of entry to a group even where posts do not actively promote such behaviour.

\section{Strengths and limitations}

This study is the first of its kind to explore images of selfharm across a number of social media platforms. We were not restrictive in terms of inclusion criteria, allowing a broad understanding of how posters themselves, presumably the majority of whom were engaging in self-harm, viewed the behaviour.

There were also a number of limitations to the study.

First, by opting to explore many images across three sites we made a trade-off between describing what was typically posted on popular social media sites and undertaking a close reading of a smaller number of images that would have allowed for deeper interpretation. Furthermore, although we studied 200 images on each platform, this is actually a small number of images compared with the many thousands of images posted about self-harm. Social media platforms are prone to rapid change so a replication study might draw different conclusions.

A second limitation was the lack of information gathered about the posters. We sampled images rather than the people who are posting about self-harm on social media. We were unable to identify whether posts about self-harm were typical for the poster, nor gather information on the self-harm of posters other than that disclosed with the image.

A further limitation is that we did not follow the discussion on posted images, from which much detail on how users respond to particular posts may be gleaned. This was a deliberate decision at the outset of the study to focus on the nature of the images posted themselves, but it could be a useful avenue for future research, particularly to explore how users respond to different types of image.

\section{Implications for research and practice}

Our findings suggest that clinicians should not be overly anxious about what is being posted about self-harm on social media. Although we found a very few posts suggesting self-injury was attractive, there were no posts that could be viewed as actively encouraging others to self-harm. Rather, the sites were being used to express difficult emotions in a variety of creative ways, offering inspiration to others through the form of texts or shared messages about recovery. There were also examples of images being shared to offer an alternative to selfharming or to offer advice to others. This should allay some of the fears of those who are concerned about social contagion and the use of social media to persuade others to self-harm. ${ }^{121631}$

There were many elements of the posts we studied that did not seem to be directly about self-harm, but about sense of identity and belonging-especially the nature of embodied distress, and the glamorisation or sexualisation of the female body. There are overlaps with online eating disorder imagery which reflect the association in young females between eating pathology and self-harm. Coupling these observations with our finding of a theme of identity and belonging, we postulate that social media posts about self-harm are being used as a vehicle for a wider exploration of key challenges in the personal and emotional life of posters. They may therefore serve a 
more useful function than has been attributed to them in recent public discussions. A recent study of the relationship between online screen time and mental well-being in adolescents suggested that moderate screen time was not intrinsically harmful and may be beneficial in an increasingly connected world. ${ }^{32}$ If there are deleterious effects, they are as likely to arise from the social isolation and lack of supportive human contact that arises from spending too much time online, as they are to be due to any specific self-harm content.

Social media are a powerful tool for peer support and communication about health and well-being issues. ${ }^{33}$ Future research could usefully explore in more detail, and in collaboration with young people who are active users of social media, these ideas about the positive and negative consequences of posting about self-harm, both for the poster and for those reading posts and responding to them.

Contributors NS conceived the study, led on the data analysis and contributed to the manuscript preparation. CB conceived the study, was involved in data analysis and preparation of the final manuscript and will act as corresponding author. $\mathrm{AH}$ conceived the study, was involved in data analysis and preparation of the final manuscript.

Funding The work was undertaken by NS in accordance with the requirements for the award of the Doctorate in Clinical Psychology at the University of Leeds.

Competing interests None declared.

Patient consent for publication Not required.

Ethics approval This research was subject to the University of Leeds ethical procedures. Ethical approval was granted by The University of Leeds SOMREC committee (MREC 15-092).

Provenance and peer review Not commissioned; externally peer reviewed.

Data sharing statement Due to copyright restrictions, we are not able to share the visual images used in this analysis.

Open access This is an open access article distributed in accordance with the Creative Commons Attribution Non Commercial (CC BY-NC 4.0) license, which permits others to distribute, remix, adapt, build upon this work non-commercially, and license their derivative works on different terms, provided the original work is properly cited, appropriate credit is given, any changes made indicated, and the use is non-commercial. See: http://creativecommons.org/licenses/by-nc/4.0/.

\section{REFERENCES}

1. Morgan C, Webb RT, Carr MJ, et al. Incidence, clinical management, and mortality risk following self harm among children and adolescents: cohort study in primary care. BMJ 2017;359:j4351.

2. Moran P, Coffey C, Romaniuk H, et al. The natural history of selfharm from adolescence to young adulthood: a population-based cohort study. Lancet 2012;379:236-43.

3. Bergen $\mathrm{H}$, Hawton $\mathrm{K}$, Waters $\mathrm{K}$, et al. Epidemiology and trends in non-fatal self-harm in three centres in England: 2000-2007. Br J Psychiatry 2010;197:493-8.

4. Madge N, Hewitt A, Hawton K, et al. Deliberate self-harm within an international community sample of young people: comparative findings from the Child \& Adolescent Self-harm in Europe (CASE) Study. J Child Psychol Psychiatry 2008;49:667-77.

5. Hawton K, Bergen H, Casey D, et al. Self-harm in England: a tale of three cities. Multicentre study of self-harm. Soc Psychiatry Psychiatr Epidemiol 2007;42:513-21.

6. Hawton K, Saunders KE, O'Connor RC. Self-harm and suicide in adolescents. Lancet 2012;379:2373-82.
7. Stallard $P$, Spears $M$, Montgomery AA, et al. Self-harm in young adolescents (12-16 years): onset and short-term continuation in a community sample. BMC Psychiatry 2013;13:328-28.

8. O'Connor RC, Rasmussen S, Miles J, et al. Self-harm in adolescents: self-report survey in schools in Scotland. Br J Psychiatry 2009;194:68-72.

9. Cooper J, Steeg S, Gunnell D, et al. Variations in the hospital management of self-harm and patient outcome: a multi-site observational study in England. J Affect Disord 2015;174:101-5.

10. Kidger J, Heron J, Lewis G, et al. Adolescent self-harm and suicidal thoughts in the ALSPAC cohort: a self-report survey in England. BMC Psychiatry 2012;12:1-12.

11. Lewis SP, Baker TG. The possible risks of self-injury web sites: a content analysis. Arch Suicide Res 2011;15:390-6.

12. Duggan JM, Heath NL, Lewis SP, et al. An examination of the scope and nature of non-suicidal self-injury online activities: implications for school mental health professionals. School Ment Health 2012;4:56-67.

13. Duffy A. Narrative Matters: You do you: teens' coconstruction of narrative, reality and identity on social media. Child Adolesc Ment Health;31.

14. Kędra J. To see more: a model for press photograph story analysis. Journal of Visual Literacy 2013;32:27-50.

15. Braun V, Clarke V. Using thematic analysis in psychology. Qual Res Psychol 2006;3:77-101.

16. Lewis SP, Heath NL, St Denis JM, et al. The scope of nonsuicidal self-injury on YouTube. Pediatrics 2011;127:e552-7.

17. Forum Qualitative Sozialforschung/Forum: Qualitative Social Research. Picturesque wounds: a multimodal analysis of self-injury photographs on Flickr. 2013.

18. Owens D, Kelley R, Munyombwe T, et al. Switching methods of selfharm at repeat episodes: Findings from a multicentre cohort study. $J$ Affect Disord 2015;180:44-51.

19. Diggins E, Kelley R, Cottrell D, et al. Age-related differences in selfharm presentations and subsequent management of adolescents and young adults at the emergency department. $J$ Affect Disord 2017;208:399-405.

20. Madge N, Hewitt A, Hawton K, et al. Deliberate self-harm within an international community sample of young people: comparative findings from the Child \& Adolescent Self-harm in Europe (CASE) study. J Child Psychol Psychiatry 2008;49:667-77.

21. Edmondson AJ, Brennan CA, House AO. Non-suicidal reasons for self-harm: a systematic review of self-reported accounts. J Affect Disord 2016;191:109-17.

22. Nixon MK, Cloutier PF, Aggarwal S. Affect regulation and addictive aspects of repetitive self-injury in hospitalized adolescents. J Am Acad Child Adolesc Psychiatry 2002;41:1333-41.

23. MacLaren VV, Best LA. Nonsuicidal self-injury, potentially addictive behaviors, and the Five Factor Model in undergraduates. Pers Individ Dif 2010;49:521-5.

24. Motz A. The psychology of female violence: crimes against the body. London: Routledge, 2008.

25. Media GR. Empowerment and the 'Sexualization of Culture' Debates. Sex Roles 2012;66:736-45.

26. Evans A, Riley S, Shankar A. Technologies of sexiness: theorizing women's engagement in the sexualization of culture. Fem Psychol 2010;20:114-31.

27. Dobson AS. Performative shamelessness on young women's social network sites: Shielding the self and resisting gender melancholia. Fem Psychol 2014;24:97-114.

28. Seko Y, Kidd SA, Wiljer D, et al. On the creative edge: exploring motivations for creating non-suicidal self-injury content online. Qual Health Res 2015;25:1334-46.

29. Gabriel F. Sexting, selfies and self-harm: young people, social media and the performance of self-development. Media International Australia 2014;151:104-12.

30. van Dijck J. Digital photography: communication, identity, memory. Visual Communication 2008;7:57-76.

31. Lewis SP, Heath NL, Michal NJ, et al. Non-suicidal self-injury, youth, and the Internet: What mental health professionals need to know. Child Adolesc Psychiatry Ment Health 2012;6:13.

32. Przybylski AK, Weinstein N. Large scale test of the Goldilocks hypothesis: quantifying the relations between digital screens and the mental well-being of adolescents. Psychological Science 2017;28:204-15.

33. Yonker LM, Zan S, Scirica CV, et al. "Friending" teens: systematic review of social media in adolescent and young adult health care. $J$ Med Internet Res 2015;17:e4. 